\title{
Correlação dos índices de agregação de Acari e Collembola com 4 fatores ambientais numa pastagem de terra firme da Amazônia.
}

\author{
Mário Dantas (') \\ Herbert O. R. Schubart $\left({ }^{2}\right)$
}

Resumo

Este trabalho trata da correlação entre os fatores temperatura do ar, umidade relativa, umidade do solo e precipitação e os índices de agregação ( $\mathrm{K}$ da binomial negativa) de população de Acari e Collembola sob Setaria sp. em pastagem rotacional e extensiva. Os artrópodes foram extraídos do solo usando-se funis de Berlese - Tullgren modificado e as coletas foram feitas de janeiro a dezembro de 1977 . Verificou-se que os índices de agregaçăo indicam uma menor agregação em Acari que em Collembola e que as correlações com os fatores físicos estudados não explicam totalmente o comportamento dos animais. Observou-se uma maior influência dos fatores estudados sobre as populações de Acari. A variável que mais influência exerce é a precipitação.

\section{INTRODUÇÃO}

Os artrópodes do solo encontram-se dispersos normalmente formando agregados, cuja distribuição possivelmente é ao acaso. Isto traz como conseqüência que, ao encontrar-se um indivíduo, haja uma grande probabilidade de serem encontrados outros. A distribuição agrupada pode ser explicada pela binomial necjativa (Dantas, 1978) e a agregação é medida pelo parâmetro $K$ (índice de agregação) da mesma binomial, calculado em função da méclia e da variância (Bliss \& Fisher, 1953). A agregação, certamente, é um fator comportamental intrínseco destas populações, porém, os fatores físicos (climáticos e edáficos) atuam também, influenciando negativa ou positivamente neste comportamento.

Alguns autores já correlacionaram fatores climáticos e edáficos com a densidade da fauna do solo, principalmente Acari e Collembola, apesar de terem chegado a resultados pouco satisfatórios. Glasgow (1939) encontrou

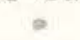

(1) - CPATU/EMBRAPA, Belém.

(2) - Instituto Nacional de Pesquisas da Amazônia, Manaus.

ACTA AMAZONICA 10(4) : 771-774. 1980 
para Acari e Collembola sob Setaria sp. nos sistemas de pastagem rotacional e extensiva. Os índices de agregação mensais são correlacionados com temperatura, umidade relativa, umidade do solo e precipitação, sendo feita para tal uma matriz de correlação simples, calculadas as regressões múltiplas e, por fim. calculada a regressão "stepwise". Para estas, usou-se um computador IBM 370 .

\section{Resultados}

Os índices de agregação $(\mathrm{K})$ são apresentados no Quadro I. Observa-se que os $\mathrm{K}$ de Acari são mais altos do que os de Collembola, indicando que a agregação de Acari é mais baixa. Verifica-se uma flutuação nos K, apresentando-se menores índices em abril e agosto/setembro, o que corresponde aproximadamente aos períodos mais chuvosos e mais seco respectivamente.

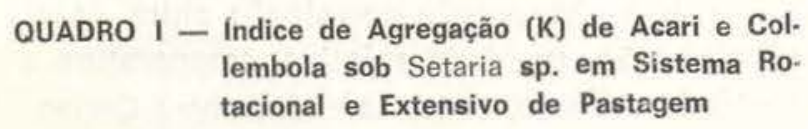
lembola sob Setaria sp. em Sistema Rotacional e Extensivo de Pastagem

\begin{tabular}{|c|c|c|c|c|}
\hline & \multicolumn{2}{|c|}{ ACARI } & \multicolumn{2}{|c|}{ COLLEMBOLA } \\
\hline & \multicolumn{4}{|c|}{ Pastagem } \\
\hline & Rotacional & Extensiva & Rotacional & Extensiva \\
\hline & K1 & K3 & K2 & K4 \\
\hline Janeiro & 1,8 & - & 0,9 & - \\
\hline Fevereiro & 3,9 & 1,8 & 1,3 & 2,6 \\
\hline Março & 2,6 & 1.4 & 0,9 & 2,0 \\
\hline Abril & 0,6 & 0,7 & 0,2 & 0,3 \\
\hline Maio & 1.9 & 1,6 & 0.7 & 0,5 \\
\hline Junho & 1.9 & 2,6 & 1,5 & 1,5 \\
\hline Julho & 1,8 & 0,8 & 1,1 & 0,5 \\
\hline Agosto & 0.4 & 1,3 & 0,2 & 0,5 \\
\hline Setembro & 0,3 & 0,6 & 0,5 & 0,5 \\
\hline Outubro & 1.3 & 1,8 & 0,9 & 1,0 \\
\hline Novembro & 2,0 & 0,8 & 0,3 & 0,3 \\
\hline Dezembro & 5,2 & 12,3 & 0,9 & 2,5 \\
\hline
\end{tabular}

Os fatores físicos aqui considerados encontram-se no Quadro II. Os dados de umidade do solo se referem somente a nove meses.
OUADRO II - Temperatura, Umidade Relativa, Umidade do Solo e Precipitação. Dados coletados durante 1977, na Regiáo de Itacoatiara

\begin{tabular}{lcccc}
\hline & $\begin{array}{c}\text { X1 } \\
\text { Temperatura }\end{array}$ & $\begin{array}{r}\text { X2 } \\
\text { U.R. }\end{array}$ & $\begin{array}{r}\text { X3 } \\
\text { U.Solo }\end{array}$ & $\begin{array}{c}\text { X4 } \\
\text { Precipitação }\end{array}$ \\
\hline Janeiro & 26,1 & 87,0 & - & 232,5 \\
Fevereiro & 25,3 & 88,0 & - & 222,4 \\
Março & 25,0 & 87,0 & - & 375,9 \\
Abril & 26,1 & 88,0 & 18,3 & 248,2 \\
Maio & 29,8 & 88,0 & 13,3 & 278,6 \\
Junho & 25,8 & 86,0 & 18,7 & 169,2 \\
Julho & 26,3 & 82,0 & 9,2 & 86,2 \\
Agosto & 27,3 & 75,0 & 7,8 & 28,7 \\
Setembro & 27,5 & 75,0 & 24,1 & 94,7 \\
Outubro & 26,8 & 80,0 & 20,8 & 135,9 \\
Novembro & 27,2 & $80,0+$ & 27,3 & 44,7 \\
Dezembro & 26,2 & $83,0+$ & 23,9 & 387,1 \\
\hline
\end{tabular}

$(+)$ - Em falto dos dados, foi usada a média de 9 anos anteriores.

Os coeficientes de correlação simples apresentados no Quadro III mostram a existência de uma correlação negativa entre temperatura e o índice de agregação de Collembola, no sistema extensivo, e ainda a existência de correlações positivas entre precipitação e os índices de agregação de Acari, nos sistemas rotacional e extensivo, e de Collembola no sistema extensivo. Umidade relativa e umidade do solo não apresentaram correlação com os índices de agregação.

QUADRO III - Coeficientes de Correlaçäo Simples Entre os K e Temperatura (X1), U.R. (X2), Umidade do Solo (X3) e Precipitaçāo (X4)

\begin{tabular}{lrrrl}
\hline & X1 & X2 & X3 & \multicolumn{1}{c}{ X4 } \\
\hline K1 & $-0,35$ & 0,29 & 0,31 & $0,63^{* *}$ \\
K2 & $-0,43$ & 0,42 & $-0,05$ & 0,29 \\
K3 & $-0,14$ & $-0,10$ & 0,29 & $0,56^{*}$ \\
K4 & $-0,60^{* *}$ & 0,31 & 0,29 & $0,65^{* *}$ \\
\hline
\end{tabular}

$\left({ }^{*}\right)-p<0,10$.

(**) $-p<0,05$. 
A regressão múltipla mostra $R^{2}$ significativo, a $5 \%$, apenas para $\mathrm{K} 3$, apresentando-se as variações $\mathrm{X} 2$ (UR) e $\mathrm{X} 4$ (precipitação) com maior interferência no modelo. Para os demais
$K$ näo se mostraram significativos os $R^{2}$. $A$ seguir, são dadas as equações resultantes da análise de regressão múltipla:

$$
\begin{aligned}
& \mathrm{K} 1=11,6528-0,1948 \mathrm{X}_{1}-0,0820 \mathrm{X}_{2}+0,0209 \mathrm{X}_{3}+0,0100 \mathrm{X}_{4} \mathrm{R}^{2}=0,56 \\
& \begin{array}{llll}
(0,411) & (0,120) & (0,077) & (0,005) \quad N=9
\end{array} \\
& \mathrm{~K} 2=2,5578-0,1262 \mathrm{X}_{1}+0,0195 \mathrm{X}_{2}-0,0075 \mathrm{X}_{3}+0,0005 \mathrm{X}_{4} \mathrm{R}^{2}=0,20 \\
& \begin{array}{lll}
(0,167) & (0,048) \quad(0,031) & (0,002) \quad N=9
\end{array} \\
& \mathrm{~K} 3=54,8398-0,7592 \mathrm{X}_{1}-0,4535 \mathrm{X}_{2}-0,0118 \mathrm{X}_{3}+0,0328 \mathrm{X}_{4} \mathrm{R}^{2}=0,85^{*} \\
& \begin{array}{llll}
(0,606) & (0,177) & (0,113) & (0,007) \quad \mathrm{N}
\end{array}=9 \\
& \mathrm{~K} 4=11,7845-0,2321 \mathrm{X}_{1}-0,0681 \mathrm{X}_{2}-0,0002 \mathrm{X}_{3}+0,005 \mathrm{X}_{4} \mathrm{R}^{2}=0,72 \\
& (0,162) \quad(0,047) \quad(0,030) \quad(0,001) \quad N=9
\end{aligned}
$$

Como se vê, $\mathrm{K} 1, \mathrm{~K} 3$ e $\mathrm{K} 4$, as variáveis em estudo contribuem com mais de $50 \%$ para a explicação do fenômeno, sendo que para K2 a porcentagem é muito baixa.

Os resultados da análise de regressão "stepwise" mostram que as variáveis que mais contribuem para a explicação da agregação dos artrópodes do solo aqui estudada são, X4, X2 \& X1, pela ordem decrescente. Os dados resultantes da análise são os que se seguem :

$$
\begin{array}{ll}
\mathrm{K} 1=0,3454+0,008 \mathrm{X}_{4} & \mathrm{R}^{2}=0,46 * \\
\mathrm{~K} 2=3,7619-0,113 \mathrm{X}_{1} & \mathrm{R}^{2}=0,09 \\
\mathrm{~K} 3=34,6827-0,460 \mathrm{X}_{2} & \mathrm{R}^{2}=0,79 \text { * } \\
\mathrm{K} 4=0,2062-0,004 \mathrm{X}_{4} & \mathrm{R}^{2}=0,41 \\
{ }^{\mathrm{p}}<0,05 \quad \text { "* } \mathrm{p}<0,01
\end{array}
$$

Por estes dados, vê-se que Acari tem seu comportamento agregacional mais atingido pelos fatores físicos do que Collembola. Também se verifica que Acari sob Setaria, no sistema extensivo de pastagem, são mais atingidos e ainda que precipitação $(X 4)$ é o fator que exerce maior influência. Como se pode ver, os resultados são condizentes, isto é, uma análise confirma a outra.

\section{DISCUSSÃo}

Os fatores considerados não explicam totalmente o comportamento agregacional das artrópødes do solo, aliás, como seria de esperar-se. O que era esperado e está confirmado pelos resultados obtidos é que os fatores fí- sicos exercem influências sobre as populações de artrópodes do solo, atuando sobre estes, ora como limitantes, ora como estimulantes às condições de vida. Logicamente, há outros fatores que carecem de consideração se se desejar um modelo capaz de explicar o comportamento dos artrópodes do solo. Entre esses, poderiam ser citados textura do solo, matéria orgânica, cobertura florística, temperatura do solo e fatores biológicos de comportamento intrínsecos aos próprios animais, que necessitam de ser melhor estudados.

Chama-nos a atenção o fato de que a um "stress" do ambiente físico segue-se uma queda na densidade da população, conforme se vê pelos dados de Dantas (1978), e um aumento no indice de agregação. Isto leva-nos a deduzir que um "stress" físico reduz o ambiente propício à vida dos artrópodes, pelo que estes são levados a uma agregação mais acentuada. Agora com relação à distribuição dos agregados, supõe-se que seja ao acaso dependendo dos micro-habitats. O tamanho destes agregados também é um assunto relacionado com os micro-habitats além das condições reprodutivas da população, pois é possível haver ambiente disponivel e não totalmente ocupado pela população dada a sua incapacidade em ocupá-lo.

Diante dos resultados, consideramos boa a participação dos fatores em estudo na explicação do comportamento agregacional de AcaIi e Collembola, já que chega a um coeficiente $\left(R^{2}\right)$ em torno de $50 \%$. Collembola apresen. ta mais baixos coeficientes $\left(R^{2}\right)$ talvez pelo fa- 
to de ter normalmente maior agregação do que Acari, tendo, deste modo, seu comportamento agregacional menos atingido pelos fatores físicos. Agora, é preciso ter em mente que, para tal comportamento ser melhor entendido tem que ser considerado, principalmente, o ciclo de vida dos animais, pois o período de eclosão com o aparecimento de novos indivíduos deve ter influência marcante no indice de agregação.

Observa-se que, no sistema rotacional onde ccorre maior pisoteio instantâneo, os quatro fatores considerados não explicam suficientemente a agregação como se pode inferir dos $R^{2}$ das análises de regressão múltipla e "stepwise".

\section{CONCLUSÃO}

Diante das evidências que se apresentaram, conclui-se que os fatores temperatura do ar, umidade do ar e do solo e precipitação não explicam totalmente os índices de agregação de Acari e Collembola buscando-se novos fatores como matéria orgânica, textura e temperatura do solo e fatores biológicos para participarem do modelo. Os fatores estudados €xercem maior influência sobre as populações de Acari do que sobre as de Collembola, apesar de estas terem maior agregação. É provável que em Collembola os fatores biológicos sejam mais importantes do que em Acari.

\section{AGRADECIMENTOS}

Ao Dr. Enedino Corrêa da Silva do D.M.Q. - EMBRAPA pela programação e análise dos dados.

\section{SUMMARY}

This paper deals with the correlations of aggregation indices ( $\mathrm{K}$ from negative binomial) of Acari and Collembola populations under Setaria sp., in rotational and extensive pastures versus other factors, such as: air temperature, relative humidity, moisture and precipitation. The Arthropods were extracted from soil by modified Beriese - Tullgren funnels and the samples were collected from January to December, 1977. It was verified that aggregation indices show smaller aggre. gation in Acari than in Collembola. Correlation indices and studied physical factors do not explain completelly animal behaviour. It was observed that studied factors have major influence upon Acari populations than ColIembola, and the variable of more influence is precipitation.

\section{BIBLIOGRAFIA}

BLISS, C.T. \& FISHER, R.

1953 - Fitting the negative binomial distribution to biological data. Biometrics, 9: 176-200.

DANTAS, M.

1978 - Pastagem da Amazônia Central : Ecologia e Fauna do Solo. Tese de mestrado apresentada à FUA/INPA em janeiro de 1978. INPA, Manaus, AM.

Dhillon, B.S. \& GrBSon, N.H.C.

1962 - A study on the Acarina and Collembolan of agricultural soils. 1. Numbers and distribution in undisturbed grassland. Pedobiologia, 1: 189-209.

GLASGOW, J.P

1939 - A population study of subterranean soil Collembola. J. Anim. Ecol., 8: 323-53.

Macmillan, J.H.

1969 - The ecology of the Acarine and Collembo. Ian fauna of two New Zeland pastures. Pedobiologia, 9 (5/6): 372-404, apud Bio. Abst. 11537, 1970.

SChuBaRT, H.O.R.; DANTAS, M. \& ANTONY, L.M.M.K.

s.d. - Descrição de uma modificação econômica do funil de Berlese - Tullgren para proscessamento de muitas unidades de amostra simultâneas. Acta Amazonica (em preparação).

SINGH, U.R.

1975 - A correlation study between Collembolan population and moisture gradient at varanasi forest, India. Int. J. Ecol. Environ. Sci., 1 (3): 179-80. apud Bio. Abst., 31415, 1977.

(Aceito para publicação em $23 / 10 / 78$ ) 